\title{
Évaluation du BactoScan FC pour la numération des bactéries du lait cru
}

\author{
Véronique NINANE ${ }^{\mathrm{a} *}$, Koen DE REU ${ }^{\mathrm{b}}$, Robert OGER ${ }^{\mathrm{c}}$, \\ Wim REYBROECK ${ }^{\mathrm{b}}$, André GUYOT ${ }^{\mathrm{a}}$ \\ ${ }^{a}$ Département qualité des productions agricoles, Centre de Recherches Agronomiques, \\ chaussée de Namur 24, 5030 Gembloux, Belgique \\ ${ }^{\mathrm{b}}$ Departement kwaliteit van dierlijke producten en transformatietechnologie, \\ Centrum voor Landbouwkundig Onderzoek, Brusselsesteenweg 370, 9090 Melle, Belgique \\ ${ }^{\mathrm{c}}$ Section biométrie, gestion des données et agrométéorologie, \\ Centre de Recherches Agronomiques, rue de Liroux 9, 5030 Gembloux, Belgique
}

(Reçu le 27 décembre 1999; accepté le 27 avril 2000)

\begin{abstract}
Evaluation of BactoScan FC for the enumeration of bacteria in raw milk. The evaluation of BactoScan FC for routine enumeration of bacteria in raw milk was carried out according to the model proposed by the International Dairy Federation. The linearity of response of BactoScan FC was verified between 6000 and 27000000 pulses $\cdot \mathrm{mL}^{-1}$ without reaching the limits of detection which have not been determined. The transmission error was estimated at $0.09 \%$ and the repeatability at $0.084 \log _{10} \mathrm{CFU} \cdot \mathrm{mL}^{-1}$ for a $60000 \mathrm{CFU} \cdot \mathrm{mL}^{-1}$ mean level of contamination. The stability of the equipment over time was verified; in both the short term and the long term, reproducibility is in the same order of magnitude as repeatability. The relationship between BactoScan FC and the reference method is linear over the whole range analysed ( 700 to $26000000 \mathrm{CFU} \cdot \mathrm{mL}^{-1}$ ), with a determination threshold estimated at under $4000 \mathrm{CFU} \cdot \mathrm{mL}^{-1}$. The regression equation between the reference method and the BactoScan FC is: $\log _{10}\left(\mathrm{CFU} \cdot \mathrm{mL}^{-1}\right)=0.839 \times \log _{10}\left(\right.$ pulses $\left.\cdot \mathrm{mL}^{-1}\right)$ +0.095 . The accuracy of estimation of the reference value, expressed by the residual standard deviation of the regression, is $0.287 \log _{10}$ CFU.mL $\mathrm{m}^{-1}$. Finally, the independence of this estimate compared to the somatic cell level of the samples was verified.
\end{abstract}

\section{BactoScan FC / bacteriological quality / raw milk}

Résumé - L'évaluation du BactoScan FC pour la numération en routine des bactéries du lait cru a été réalisée selon le modèle proposé par la Fédération Internationale de Laiterie. La linéarité de réponse du BactoScan FC a été vérifiée entre 6000 et 27000000 impulsions. $\mathrm{mL}^{-1}$ sans toutefois atteindre les limites de détection qui restent indéterminées. L'erreur de transfert a été estimée

* Correspondance et tirés-à-part

Tél. : (32) 081620 361; fax : (32) 081620388 ; e-mail : ninane @ cragx.fgov.be 
à $0,09 \%$ et la répétabilité à $0,084 \log _{10} \mathrm{UFC} \cdot \mathrm{mL}^{-1}$ pour un niveau de contamination moyen de $60000 \mathrm{UFC} \cdot \mathrm{mL}^{-1}$. La stabilité de l'appareil dans le temps a été vérifiée ; tant à court terme qu'à long terme, sa reproductibilité est du même ordre de grandeur que sa répétabilité. La relation entre le BactoScan FC et la méthode de référence est linéaire sur toute la plage analysée (de 700 à $26000000 \mathrm{UFC} \cdot \mathrm{mL}^{-1}$ ), avec un seuil de détermination estimé à moins de $4000 \mathrm{UFC} \cdot \mathrm{mL}^{-1}$. L'équation de la régression entre la méthode de référence et le BactoScan FC est : $\log _{10}$ $\left(\right.$ UFC $\left.\cdot \mathrm{mL}^{-1}\right)=0,839 \times \log _{10}$ (impulsions $\left.\cdot \mathrm{mL}^{-1}\right)+0,095$. La justesse de l'estimation de la valeur de référence, exprimée par l'écart type résiduel de la régression, est de $0,287 \log _{10} \mathrm{UFC} \cdot \mathrm{mL}^{-1}$. Enfin, l'indépendance de cette estimation par rapport au taux de cellules somatiques des échantillons a été vérifiée.

\section{BactoScan FC / qualité bactériologique / lait cru}

\section{INTRODUCTION}

Le dénombrement de la flore du lait cru dans le cadre du paiement du lait au producteur est aujourd'hui effectué à l'aide de méthodes instrumentales rapides, toutes calibrées par rapport à une méthode de référence, et agréées, en Belgique, par le Ministère de l'Agriculture. Pour pouvoir être exploitées dans ce contexte, les méthodes instrumentales doivent en effet présenter des caractéristiques instrumentales et analytiques leur permettant de se substituer au mieux à la méthode de référence, l'agrément en est le garant.

La société Foss Electric DK, qui, avec le BactoScan, a développé la méthode instrumentale de détermination de la flore totale dans le lait cru la plus répandue dans les laboratoires européens, propose aujourd'hui un nouveau modèle d'appareil : le BactoScan FC. Cette nouvelle génération d'appareils offre une plus grande capacité d'analyse, une plus grande simplicité d'utilisation et de meilleures performances analytiques que les versions précédentes. Au niveau méthodologique, le BactoScan FC diffère des anciennes versions, notamment, par le mode de traitement des composés du lait susceptibles d'interférer avec le comptage des bactéries, par le marqueur cellulaire employé et par la méthode de séparation des unités bactériennes (cytométrie de flux). Enfin, au niveau informatique, le BactoScan FC est équipé d'un logiciel d'utilisation et de traitement des données commun aux autres appareils proposés par Foss Electric DK pour l'analyse du lait.

L'objet de ce travail est d'évaluer les performances instrumentales et analytiques de ce nouveau type de BactoScan en vue de répondre à une demande d'agrément.

\section{MATÉRIEL ET MÉTHODES}

Les essais ont commencé à la fin de février 1999 et se sont achevés au début de juin 1999.

\subsection{Dénombrement de la flore totale du lait cru}

\subsubsection{Méthode de référence}

La préparation des échantillons de lait et des dilutions en vue de l'examen microbiologique a été réalisée selon les recommandations de la norme FIL 122C : 1996 [7], en prenant comme diluant une solution de Ringer diluée au quart. La norme a été modifiée au niveau de l'homogénéisation de la première dilution qui a été réalisée, comme celle des dilutions suivantes, à l'aide d'un agitateur mécanique de type « vortex » plutôt que manuellement. Les germes ont été 
dénombrés par la méthode de comptage des colonies à $30{ }^{\circ} \mathrm{C}$ décrite dans la norme FIL 100B : 1991 [4]. Elle a été appliquée en utilisant, pour l'ensemencement, le milieu complet déshydraté « Agar Plate-Count au lait écrémé » (Merck).

La répétabilité de la méthode de référence a été évaluée, selon le modèle proposé par la norme FIL 128 : 1985 [3], à partir de 53 échantillons de lait provenant de tanks de producteurs. Le lait, maintenu entre 0 et $4{ }^{\circ} \mathrm{C}$ dans les tanks de producteurs, provient de 4 ou, le plus souvent, de 6 traites.

\subsubsection{BactoScan FC}

Le BactoScan FC (Foss Electric, Hillerød, Danemark) est un instrument automatisé de comptage rapide des bactéries du lait cru. Il analyse un échantillon en $9 \mathrm{~min}$, à une cadence, pour le modèle évalué, de 150 échantillons par heure. Le fonctionnement du BactoScan FC est illustré à la Figure 1.

Après homogénéisation, le lait est incubé avec une solution tamponnée de bromure d'éthidium, de détergent et d'enzyme protéolytique pour réduire et disperser les constituants susceptibles d'interférer avec le comptage des bactéries (globules gras, protéines, cellules somatiques, ...) et, en même temps, pour marquer les cellules bactériennes. Pendant l'incubation, des passages répétés du mélange lait - solution d'incubation au travers d'un tamis de $0,22 \mu \mathrm{m}$ assurent l'homogénéisation du mélange et contribuent à disperser les constituants.

Un échantillon du mélange est ensuite entraîné par une solution tamponnée de

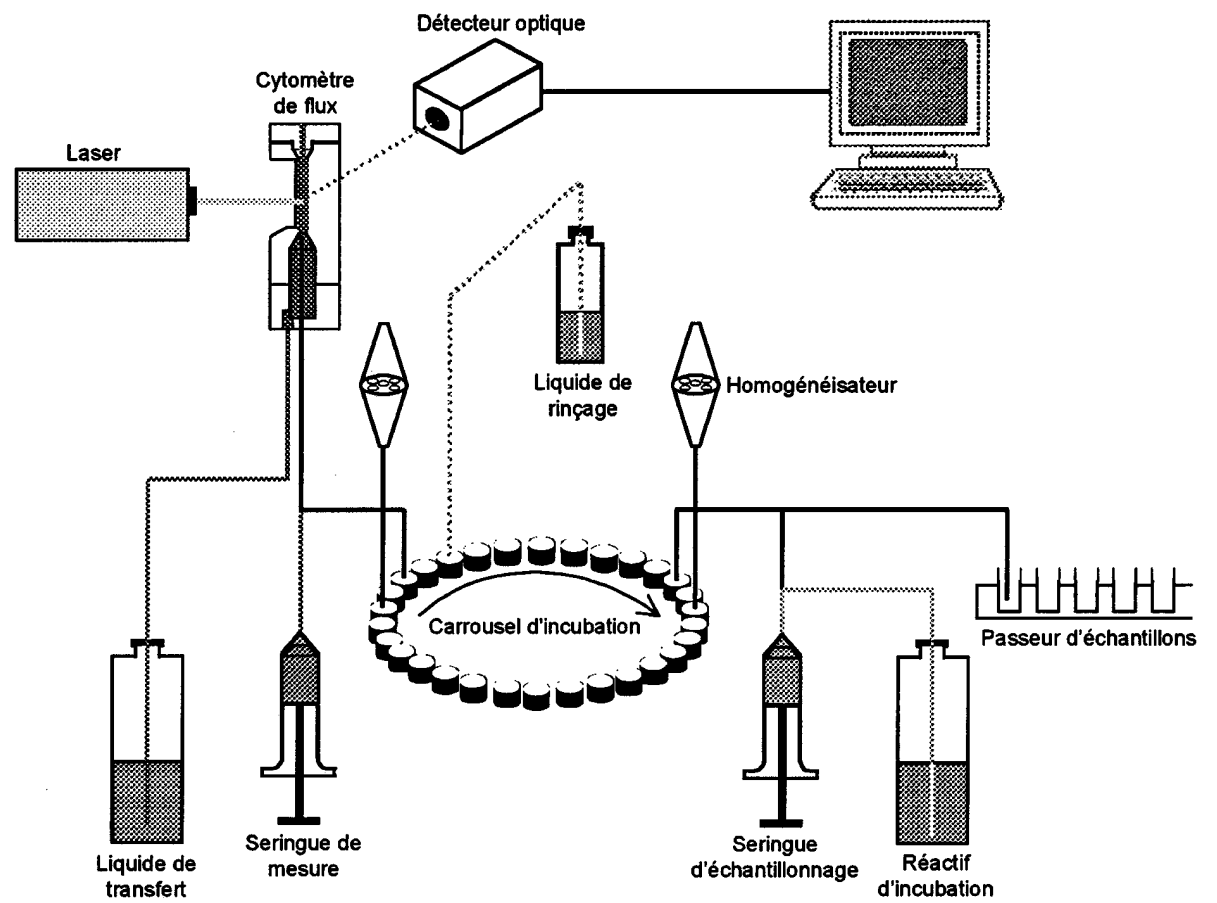

Figure 1. Schéma de fonctionnement du BactoScan FC (adapté d'après Foss Electric, Hillerød, Danemark).

Figure 1. Operation outline of the BactoScan FC (adapted from Foss Electric, Hillerød, Danemark). 
détergent vers l'unité de lecture. Les cellules bactériennes, marquées par le bromure d'éthidium, passent en flux laminaire devant un lecteur optique. Excité par un faisceau laser, le bromure d'éthidium émet un rayonnement de fluorescence. La lumière émise est détectée par le lecteur optique qui génère une impulsion électronique au passage de chaque bactérie. Les impulsions sont comptées et converties en unités de référence (UFC. $\mathrm{mL}^{-1}$ ) à l'aide d'une table de conversion déterminée par le laboratoire. Le BactoScan FC doit en effet être calibré par rapport à la méthode de référence.

Le système est rincé, entre les échantillons, avec un liquide de rinçage et, en fin de journée, avec une solution d'ammoniaque $(0,12 \%)$.

\subsection{Détermination du taux de cellules somatiques}

Le taux de cellules somatiques des échantillons de lait a été déterminé par une méthode fluoro-opto-électronique (Fossomatic 5000 développé par Foss Electric, Hillerød, Danemark) dont l'exactitude est régulièrement vérifiée par des tests analytiques comparatifs belges et internationaux : IDF collaborative test « somatic cell counting in milk » organisé par le «Bundesanstalt für Milchforschung » de Kiel (Allemagne) et étude interlaboratoire organisée par CECALAIT sous le patronage d'ICAR à Poligny (France).

\subsection{Préparation des échantillons stabilisés}

Les échantillons stabilisés sans conservateur ont été préparés par congélation immédiate à $-20{ }^{\circ} \mathrm{C}$ d'un lait réparti en sous-échantillons. La teneur en germes du lait a préalablement été augmentée par ajout de $1,3 \%$ d'un lait à très haute teneur en germes $\left(=8000000 \mathrm{UFC} \cdot \mathrm{mL}^{-1}\right)$.

Les échantillons stabilisés avec conservateur ont été préparés à partir d'un lait addi- tionné de lactose (7 \% en concentration finale), de yogourt $(0,1$ à $0,3 \%$ pour obtenir une teneur en germes d'environ $50000 \mathrm{UFC} \cdot \mathrm{mL}^{-1}$ ) et d'azoture de sodium $(0,03 \%)$. Les échantillons ont été maintenus entre 2 et $4{ }^{\circ} \mathrm{C}$ pendant $2 \mathrm{j}$ avant d'être congelés à $-20^{\circ} \mathrm{C}$, pour que leur teneur en germes atteigne un niveau proche du seuil de pénalité (100 $\left.000 \mathrm{UFC} \cdot \mathrm{mL}^{-1}\right)$.

Les échantillons stabilisés lyophilisés ont été préparés à partir d'un lait partiellement évaporé (1/4) par chauffage au bain-marie à $45^{\circ} \mathrm{C}$ sous pression réduite $(0,9$ bar $)$. Le lait évaporé a été refroidi à $4{ }^{\circ} \mathrm{C}$ puis réparti en sous-échantillons de $30 \mathrm{~mL}$ et congelé à $-20{ }^{\circ} \mathrm{C}$. Les échantillons congelés ont été lyophilisés sous vide $\left(10^{-2} \mathrm{mbar}\right)$ et conservés au frais $\left(4{ }^{\circ} \mathrm{C}\right)$.

\section{4. Évaluation du BactoScan FC}

\subsubsection{Linéarité de réponse}

La linéarité de la réponse a été évaluée au début et à la fin de la période d'essais. L'évaluation a été effectuée, chaque fois, à partir de 2 laits provenant de producteurs différents. La teneur en germes des laits a été augmentée par ajout de 20 à $25 \%$ d'un lait à très haute teneur en germes $\left(\simeq 8000000 \mathrm{UFC} \cdot \mathrm{mL}^{-1}\right)$. Les gammes ont été obtenues par dilutions des laits « dopés » avec les laits originaux, pour les valeurs élevées ( $\geq 1500000 \mathrm{impulsions} \cdot \mathrm{mL}^{-1}$ ), et par dilutions des laits originaux avec de l'eau stérile, pour les valeurs plus petites $\left(\leq 1500000\right.$ impulsions $\left.\cdot \mathrm{mL}^{-1}\right)$.

\subsubsection{Erreur de transfert}

L'erreur de transfert d'un échantillon sur le suivant a été évaluée à partir de 3 séries de 20 échantillons de lait affichant de 50000 à plus de 20000000 impulsions.mL ${ }^{-1}$, soit un taux de germes s'échelonnant d'environ 10000 à plus de $1700000 \mathrm{UFC} \cdot \mathrm{mL}^{-1}$. L'analyse des 3 séries a été répartie sur la durée de l'évaluation de l'appareil. 
Les échantillons de lait ont été analysés en alternance avec le liquide de transfert du BactoScan FC (solution tamponnée de détergent) selon la succession :

lait (L) - lait (L') - liquide de transfert (B) - liquide de transfert (B').

L'erreur de transfert a été calculée selon l'équation [12] :

Erreur de transfert $\%=\frac{\Sigma \mathrm{B}-\Sigma \mathrm{B}^{\prime}}{\Sigma \mathrm{L}^{\prime}} \times 100$.

\subsubsection{Répétabilité}

La répétabilité a été évaluée à partir de 337 échantillons répartis en 3 séries analysées au début, en cours et à la fin de la période d'essais. L'analyse en double a été réalisée par séries de 18 échantillons (double passage des porte-échantillons). L'écarttype de répétabilité $\left(\mathrm{s}_{\mathrm{r}}\right)$ et la répétabilité $(\mathrm{r})$ ont été calculés, à partir des données transformées en UFC· $\mathrm{mL}^{-1}$ à l'aide de l'équation de calibrage (point 3.2.3), selon le modèle proposé par la norme FIL 128 : 1985 [3]. L'écart-type relatif géométrique a été calculé selon la formule :

$\operatorname{GRSD}_{\mathrm{r}}\left(\% \mathrm{UFC} \cdot \mathrm{mL}^{-1}\right)=\left(10^{\mathrm{Sr}}-1\right) \times 100$.

\subsubsection{Stabilité au cours du temps}

La stabilité sur une courte durée (6 h) a été évaluée en début et en fin de période d'essais à partir de laits sous-échantillonnés et maintenus à $4{ }^{\circ} \mathrm{C}$. Au total, 4 laits ont été analysés : 2 à taux de contamination proche du seuil de pénalité $\left(100000 \mathrm{UFC} \cdot \mathrm{mL}^{-1}\right)$, un à faible niveau de contamination $\left(<10000 \mathrm{UFC} \cdot \mathrm{mL}^{-1}\right)$ et un à niveau de contamination élevé (> $\left.250000 \mathrm{UFC} \cdot \mathrm{mL}^{-1}\right)$.

La stabilité sur une longue durée (de 6 à 10 semaines) a été évaluée à partir des 3 types d'échantillons stabilisés (lait congelé, lait congelé avec conservateur et lait lyophilisé reconstitué avec de l'eau stérile). Au total, 8 échantillons lyophilisés, 12 échantillons congelés sans conservateur et
12 échantillons congelés avec conservateur ont été analysés de 3 à 5 fois au cours de cette période.

Les paramètres de stabilité ont été directement estimés à partir des données transformées en UFC. $\mathrm{mL}^{-1}$ à l'aide de l'équation de calibrage donnée au point 3.2.3. L'écart-type de reproductibilité $\left(\mathrm{s}_{\mathrm{R}}\right)$ a été estimé à partir de la variance de la population. Pour la stabilité à court terme, les souséchantillons de 2 laits ont été analysés en double. Dans ces deux cas, l'écart-type de reproductibilité a été calculé à l'aide du modèle proposé dans la norme FIL 135B : 1991 [5]. La reproductibilité (R) a été estimée selon le modèle proposé par la norme FIL 128 : 1985 [3] et l'écart-type relatif géométrique $\left(\mathrm{GRSD}_{\mathrm{R}}\right)$ a été calculé en remplaçant $S_{r}$ par $S_{R}$ dans la formule donnée au point 2.4.3.

\subsubsection{Caractéristiques analytiques}

Les paramètres analytiques du BactoScan FC ont été estimés, après élimination de 4 valeurs aberrantes sur base d'une analyse des résidus de la régression (niveau d'élimination $=3,29$ ), à partir de 508 échantillons de lait provenant essentiellement de tanks de producteurs (échantillons représentant le tout venant habituellement analysé par le laboratoire). La plupart des échantillons ont été analysés sans interruption de la chaîne de froid ; certains ont toutefois préalablement fait l'objet d'autres déterminations nécessitant une augmentation temporaire de la température. Leur teneur en germes, qui s'échelonne de 700 à $26200000 \mathrm{UFC} \cdot \mathrm{mL}^{-1}$, a été déterminée une fois par chacune des méthodes (référence et BactoScan FC). Les analyses, réalisées par séries de 20 échantillons, ont été réparties tout au long de la période d'essai.

La précision de l'appareil a été calculée en multipliant l'écart-type résiduel $\left(\mathrm{s}_{\mathrm{y}, \mathrm{x}}\right) \mathrm{de}$ la régression de calibrage par la valeur critique de la variable $t$ de Student pour 500 degrés de liberté et pour un risque de première espèce de $5 \%(1,965)$. 


\section{RÉSULTATS}

\subsection{Caractéristiques instrumentales}

\subsubsection{Linéarité de réponse}

Les régressions linéaires, calculées pour chaque lait, entre les valeurs théoriques et les valeurs observées sont présentées à la Figure 2. Les graphiques montrent que la réponse de l'appareil est linéaire sur toute la plage analysée $(\mathrm{de}=2000$ à $\left.=2100000 \mathrm{UFC} \cdot \mathrm{mL}^{-1}\right)$. Portés en graphique, les écarts à la linéarité se répartissent aléatoirement autour de la droite de régression.
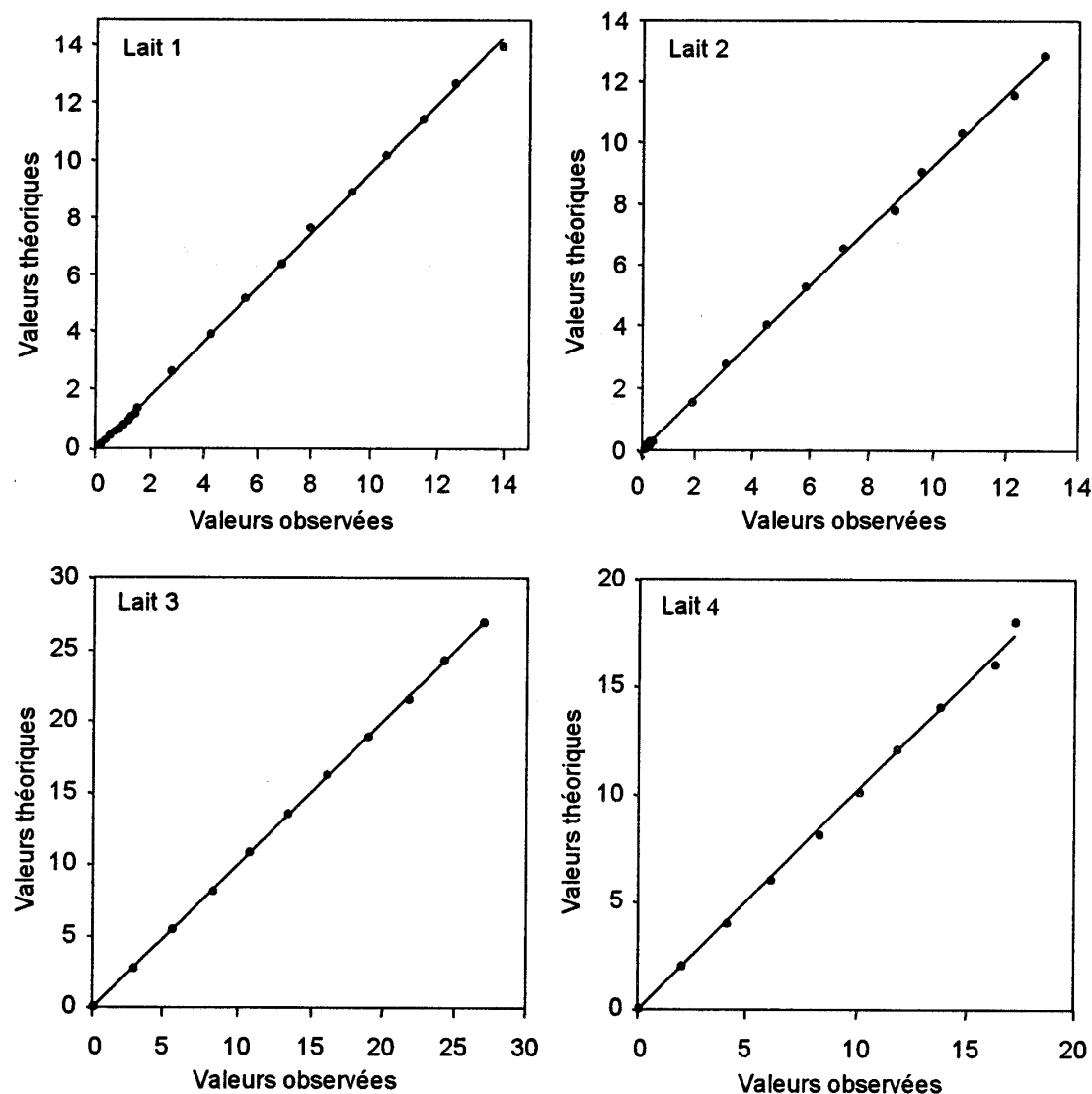

\subsubsection{Erreur de transfert}

L'erreur de transfert a été estimée à $0,09 \%$. Elle se situe en dessous de la limite annoncée par le constructeur $(<0,5 \%)$.

\subsubsection{Répétabilité}

Les paramètres de répétabilité, écart-type de répétabilité, répétabilité et écart-type relatif géométrique, du BactoScan FC sont repris dans le tableau I.

La répétabilité dépend du niveau de contamination : elle est plus faible pour des

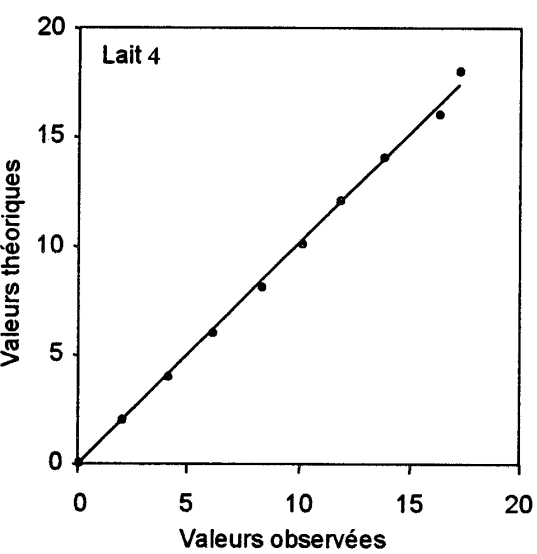

Figure 2. Linéarité de réponse du BactoScan $\mathrm{FC}$, en $10^{6}$ impulsions $\cdot \mathrm{mL}^{-1}$.

Figure 2. Linearity of response of BactoScan FC, in $10^{6}$ pulses $\cdot \mathrm{mL}^{-1}$. 
Tableau I. Répétabilité du BactoScan FC, du BactoScan 8000 et de la méthode de référence.

Table I. Repeatability of BactoScan FC, BactoScan 8000 and the reference method.

\begin{tabular}{|c|c|c|c|c|c|}
\hline & Niveau de contamination & $\mathrm{n}$ & $\mathrm{s}_{\mathrm{r}}$ & $\mathrm{r}$ & $\mathrm{GRSD}_{\mathrm{r}}$ \\
\hline Source & $\left(10^{3} \mathrm{UFC} \cdot \mathrm{mL}^{-1}\right)$ & & $\left(\log _{10}\right)$ & $\left.\mathrm{mL}^{-1}\right)$ & $(\%)$ \\
\hline BactoScan FC & & & & & \\
\hline Notre étude & $<10$ & 54 & 0,043 & 0,120 & 10,3 \\
\hline & $10-100$ & 170 & 0,031 & 0,088 & 7,5 \\
\hline & $100-250$ & 35 & 0,026 & 0,074 & 6,2 \\
\hline & $>250$ & 78 & 0,012 & 0,034 & 2,8 \\
\hline & Total & 337 & 0,030 & 0,084 & 7,1 \\
\hline BactoScan 8000 & & & & & \\
\hline Dasen et al. [1] & $<10$ & 80 & 0,132 & 0,370 & 35,5 \\
\hline & $10-15$ & 61 & 0,092 & 0,258 & 23,6 \\
\hline & $15-25$ & 88 & 0,078 & 0,218 & 19,7 \\
\hline & $25-50$ & 142 & 0,051 & 0,143 & 12,4 \\
\hline & $50-100$ & 116 & 0,035 & 0,098 & 8,4 \\
\hline & $100-250$ & 74 & 0,015 & 0,042 & 3,5 \\
\hline & $>250$ & 17 & 0,005 & 0,014 & 1,2 \\
\hline Méthode de réfe & ence & & & & \\
\hline Notre étude & $<10$ & 3 & 0,028 & 0,080 & 6,7 \\
\hline & $10-100$ & 32 & 0,044 & 0,124 & 10,6 \\
\hline & $100-250$ & 8 & 0,049 & 0,138 & 11,8 \\
\hline & $>250$ & 10 & 0,066 & 0,186 & 16,3 \\
\hline & Total & 53 & 0,049 & 0,138 & 11,9 \\
\hline
\end{tabular}

$\mathrm{n}$ : nombre d'échantillons analysés ; $\mathrm{s}_{\mathrm{r}}$ : écart-type de répétabilité $; \mathrm{r}$ : répétabilité $; \mathrm{GRSD}_{\mathrm{r}}$ : écart-type relatif géométrique de répétabilité.

$\mathrm{n}$ : number of samples analysed; $\mathrm{s}_{\mathrm{r}}$ : standard deviation of repeatability; r: repeatability ; $\mathrm{GRSD}_{\mathrm{r}}$ : geometric relative standard deviation of repeatability.

niveaux élevés $\left(\mathrm{r}=0,034 \log _{10} \mathrm{UFC} \cdot \mathrm{mL}^{-1}\right.$ pour un niveau de contamination $>250000$ $\mathrm{UFC} \cdot \mathrm{mL}^{-1}$ ) que pour des niveaux faibles $\left(\mathrm{r}=0,120 \log _{10} \mathrm{UFC} \cdot \mathrm{mL}^{-1}\right.$ pour un niveau de contamination $<10000 \mathrm{UFC} \cdot \mathrm{mL}^{-1}$ ). Pour un niveau de contamination moyen de $60000 \mathrm{UFC} \cdot \mathrm{mL}^{-1}$, elle est de $0,084 \log _{10}$ $\mathrm{UFC} \cdot \mathrm{mL}^{-1}$

\subsubsection{Stabilité au cours du temps}

Les paramètres de reproductibilité à court terme et à long terme sont repris, respectivement, dans les tableaux II et III.

La reproductibilité sur une courte durée (Tab. II) est, comme la répétabilité, plus élevée pour un faible niveau de contamination $\left(<10000 \mathrm{UFC} \cdot \mathrm{mL}^{-1}\right)$ que pour un niveau de contamination plus important.

Tant à long terme qu'à court terme, la reproductibilité est du même ordre de grandeur que la répétabilité, ce qui traduit une bonne stabilité de l'appareil dans le temps.

\subsection{Caractéristiques analytiques}

\subsubsection{Nature de la relation entre le BactoScan FC et la méthode de référence}

La relation entre la méthode de routine et la méthode de référence est, à l'examen du diagramme de dispersion (Fig. 3), proche de la linéarité pour l'ensemble des résultats. 
Tableau II. Stabilité sur une courte durée.

Table II. Short-term stability.

\begin{tabular}{lclcr}
\hline Niveau de contamination & $\mathrm{n}$ & \multicolumn{2}{c}{$\mathrm{s}$} & $\mathrm{GRSD}_{\mathrm{R}}$ \\
\cline { 3 - 4 }$\left(10^{3} \mathrm{UFC} \cdot \mathrm{mL}^{-1}\right)$ & & \multicolumn{2}{c}{$\left(\log _{10} \mathrm{UFC} \cdot \mathrm{mL}^{-1}\right)$} & $(\%)$ \\
\hline 9 & 24 & 0,050 & 0,142 & 12,3 \\
64 & 19 & $0,024^{*}$ & 0,067 & 5,6 \\
102 & 18 & $0,017^{*}$ & 0,048 & 4,0 \\
409 & 24 & 0,018 & 0,052 & 4,3 \\
\hline
\end{tabular}

* Calculés selon le modèle proposé par la norme FIL 135B : 1991 [3] ; n : nombre d'échantillons analysés ; $\mathrm{s}_{\mathrm{R}}$ : écarttype de reproductibilité ; R : reproductibilité ; $\mathrm{GRSD}_{\mathrm{R}}$ : écart-type relatif géométrique de reproductibilité.

* Calculated as proposed by the IDF standard 135B: 1991 [3]; n: number of samples analysed; $\mathrm{s}_{\mathrm{R}}$ : standard deviation of reproducibility; R: reproducibility; $\mathrm{GRSD}_{\mathrm{R}}$ : geometric relative standard deviation of reproducibility.

Tableau III. Stabilité sur une longue durée.

Table III. Long-term stability.

\begin{tabular}{|c|c|c|c|c|c|}
\hline \multirow{2}{*}{$\begin{array}{l}\text { Type d'échantillon } \\
\text { stabilisé }\end{array}$} & \multirow{2}{*}{$\begin{array}{c}\text { Niveau de } \\
\text { contamination } \\
\left(\mathrm{UFC} \cdot \mathrm{mL}^{-1}\right)\end{array}$} & \multirow[t]{2}{*}{$\mathrm{n}$} & $\mathrm{s}_{\mathrm{R}}$ & $\mathrm{R}$ & \multirow{2}{*}{$\begin{array}{c}\mathrm{GRSD}_{\mathrm{R}} \\
(\%)\end{array}$} \\
\hline & & & \multicolumn{2}{|c|}{$\left(\log _{10} \mathrm{UFC} \cdot \mathrm{mL}^{-1}\right)$} & \\
\hline Congelé sans conservateur & 68000 & 5 & 0,038 & 0,107 & 9,1 \\
\hline Congelé avec conservateur & 80000 & 4 & 0,023 & 0,065 & 5,5 \\
\hline Lyophilisé & 68000 & 3 & 0,029 & 0,082 & 6,9 \\
\hline
\end{tabular}

* Moyennes quadratiques calculées à partir de 8 ou 12 estimations ; n : nombre d'échantillons analysés ; $\mathrm{s}_{\mathrm{R}}$ : écarttype de reproductibilité ; $\mathrm{R}$ : reproductibilité $; \mathrm{GRSD}_{\mathrm{R}}$ : écart-type relatif géométrique de reproductibilité.

* Quadratic means calculated from 8 or 12 assessments; $\mathrm{n}$ : number of samples analysed; $\mathrm{s}_{\mathrm{R}}$ : standard deviation of reproducibility; R: reproducibility; $\mathrm{GRSD}_{\mathrm{R}}$ : geometric relative standard deviation of reproducibility.

L'hypothèse de linéarité a été vérifiée en appliquant le modèle d'analyse de la variance décrit dans la norme FIL 161 A : 1995 [6]. Les données ont, pour ce faire, été groupées sur base de la variable indépendante (méthode de référence), en 31 classes d'étendue croissante (de progression plus ou moins logarithmique) de manière à assurer des effectifs comparables dans chaque classe. La relation entre les valeurs moyennes calculées au sein de chaque classe est illustrée à la Figure 4 ; sa linéarité conforte l'hypothèse déduite du diagramme de dispersion (Fig. 3). Le rapport entre la somme des carrés des écarts des valeurs moyennes à la régression et la somme des carrés des écarts des valeurs individuelles au sein de chaque classe est de 1,26. Il est suffisamment petit pour que l'hypothèse de linéarité puisse être acceptée pour l'ensemble des résultats avec un degré de confiance $\alpha=0,01$. La valeur critique $\left(\mathrm{F}_{0,99}=1,74\right)$ fournie par les tables de Snedecor pour 30 et 500 degrés de liberté est en effet largement supérieure.

\subsubsection{Seuil de détermination}

Le seuil de détermination n'a pu être établi avec précision faute d'un nombre suffisant d'échantillons à très faibles teneurs en germes. On peut néanmoins considérer qu'il 


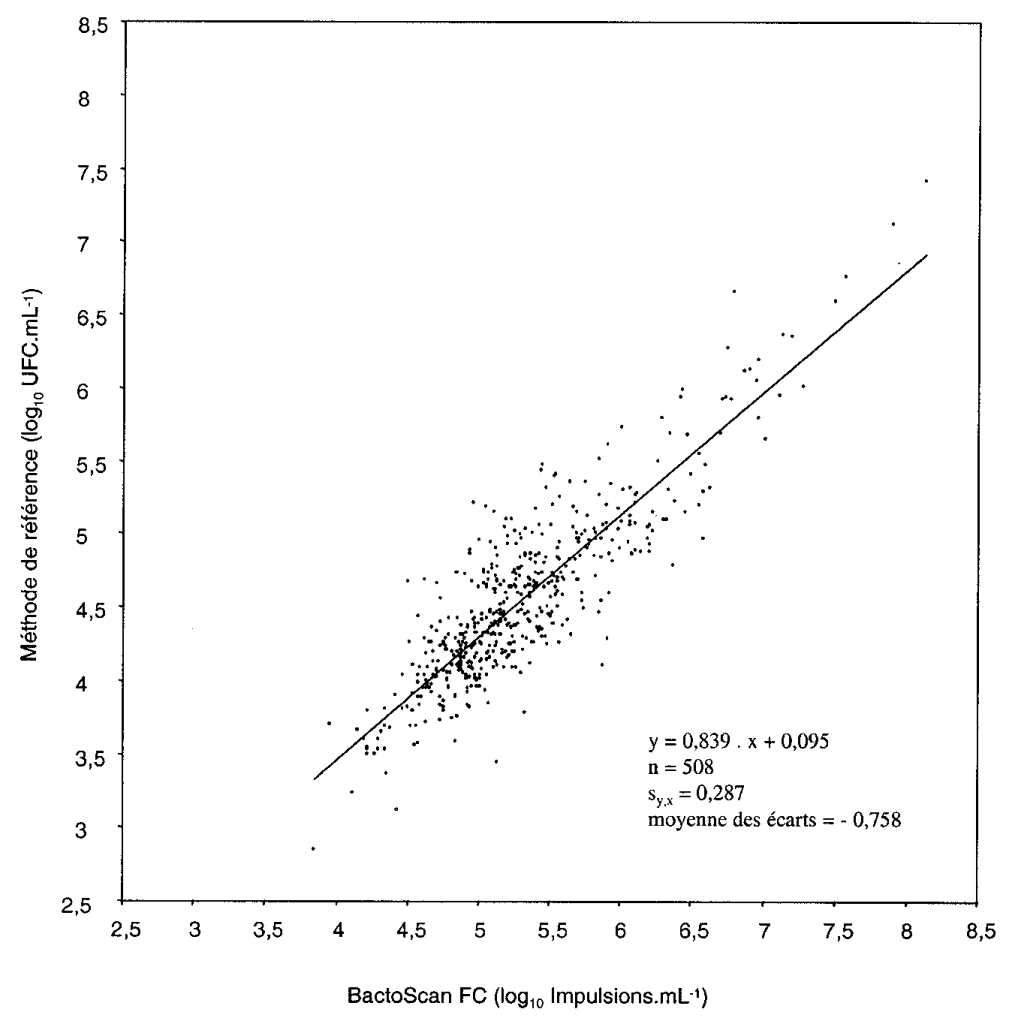

Figure 3. Diagramme de dispersion des valeurs obtenues par le BactoScan FC et par la méthode de référence.

Figure 3. Dispersion diagram for values obtained with BactoScan FC and by the reference method.

doit être inférieur à $4000 \mathrm{UFC} \cdot \mathrm{mL}^{-1}$, limite de la première classe établie pour vérifier l'hypothèse de linéarité de la relation (point 3.2.1).

\subsection{3. Équation de calibrage, justesse et précision}

En pratique, la valeur de référence est estimée à partir de la méthode de routine. L'équation de calibrage est dès lors calculée en considérant la méthode de référence comme variable dépendante et la méthode de routine comme variable indépendante. Calculée à partir de l'ensemble des échantillons, l'équation de la régression linéaire de calibrage est :

$$
\begin{gathered}
\log _{10}\left(\mathrm{UFC} \cdot \mathrm{mL}^{-1}\right)=0,839 \times \log _{10} \\
\quad\left(\text { impulsions } \cdot \mathrm{mL}^{-1}\right)+0,095 .
\end{gathered}
$$

La justesse de l'estimation de la valeur de référence, exprimée par l'écart-type résiduel $\left(\mathrm{s}_{\mathrm{y}, \mathrm{x}}\right)$ de la régression de calibrage, est de $0,287 \log _{10} \mathrm{UFC} \cdot \mathrm{mL}^{-1}$. L'écart-type résiduel est supérieur à celui annoncé par le constructeur : 0,25.

La précision d'estimation du BactoScan FC est, pour un risque d'erreur de $5 \%$, de \pm $0,564 \log _{10} \mathrm{UFC} \cdot \mathrm{mL}^{-1}$. Pour un niveau de qualité bactériologique de $100000 \mathrm{UFC} \cdot \mathrm{mL}^{-1}$, les limites de l'intervalle à l'intérieur desquelles se situe, avec une probabilité de 
$95 \%$, la valeur donnée par la méthode de référence sont, respectivement pour la limite inférieure et pour la limite supérieure, de $27000 \mathrm{UFC} \cdot \mathrm{mL}^{-1}$ et de $366000 \mathrm{UFC} \cdot \mathrm{mL}^{-1}$.

\subsubsection{Influence du taux de cellules somatiques sur l'estimation}

Le taux de cellules somatiques est un facteur susceptible d'influencer la réponse du BactoScan FC parce que le marqueur (bromure d'éthidium) se lie spécifiquement aux acides nucléiques, 1000 fois plus abondants dans les cellules diploïdes de mammifère que dans les bactéries. D'après l'étude de Suhren and Walte [10], ce facteur n'influence la réponse du BactoScan $\mathrm{FC}$ qu'au-delà d'un million de cellules $\cdot \mathrm{mL}^{-1}$, taux rarement rencontré dans la pratique. Il convient néanmoins de vérifier son influence sur l'estimation de la valeur de référence à partir de la méthode de routine. Elle a été évaluée en introduisant dans l'équation de régression linéaire les valeurs du «taux cellulaire » comme variable explicative complémentaire. L'importance de l'introduction de la variable taux cellulaire dans l'estimation de la valeur de référence a ensuite été éprouvée, d'une part, en testant la signification statistique de son coefficient angulaire et, d'autre part, en comparant la justesse des estimations obtenues avec et sans ce facteur.

L'équation de la régression linéaire, établie sur les 360 échantillons dont le taux cellulaire a été déterminé, est alors :

$\log _{10}($ référence $)=$

$$
\begin{aligned}
& 0,8240 \times \log _{10}(\text { BactoScan }) \\
& +0,0977 \log _{10} \text { (cellules) } \\
& -0,3488
\end{aligned}
$$

Le rapport $\left(\mathrm{t}_{\text {observé }}=1,77\right)$ du coefficient « taux cellulaire » $(0,0977)$ à son écart-type $(0,0551)$ est inférieur à la valeur critique $\left(\mathrm{t}_{\text {critique }}=2,24\right)$ fournie par les tables de Student pour un degré de confiance $\alpha=0,01$, permettant de considérer la variable taux cellulaire comme non significative dans l'estimation de la valeur de référence.

L'écart-type résiduel de la régression linéaire passe de 0,270 à 0,269 lorsque la variable taux cellulaire y est introduite. L'introduction de ce facteur dans la régression n'apporte donc pas d'amélioration significative de la justesse de l'estimation.

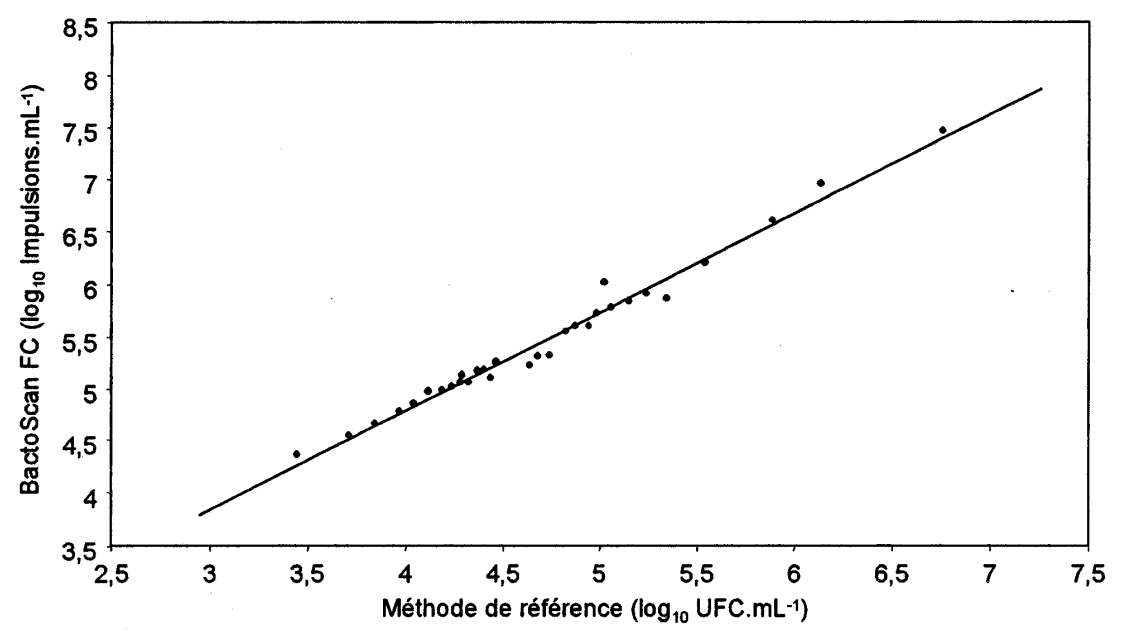

Figure 4. Relation entre les valeurs moyennes calculées au sein de chaque classe.

Figure 4. Relationship between mean values calculated in each class. 
On peut raisonnablement conclure que l'estimation de la valeur de référence à partir du BactoScan FC n'est pas influencée par le taux cellulaire, dans le contexte cellulaire rencontré dans la pratique.

\section{DISCUSSION}

Au niveau instrumental, la linéarité de réponse du BactoScan $\mathrm{FC}$ a été vérifiée entre 6000 et 27000000 impulsions $\cdot \mathrm{mL}^{-1}$ sans atteindre les limites de détection qui restent indéterminées.

L'erreur de transfert du BactoScan FC, ou la contamination d'un échantillon sur le suivant, a été estimée à $0,09 \%$. Elle est inférieure aux valeurs obtenues par Suhren and Walte [10] pour ce modèle de BactoScan : $0,22 \%$ et $0,26 \%$. Cette différence est probablement due à l'application de modèles différents d'estimation. L'étude conduite par Trossat et al. [11] fait, quant à elle, apparaître une erreur de transfert variant de $0,0 \%$ à $0,4 \%$ selon le degré de contamination de l'échantillon. L'erreur de transfert du BactoScan FC est du même ordre de grandeur que celle du BactoScan 8000 (0,16\%) [1] .

La répétabilité du BactoScan FC, comme celle du BactoScan 8000 (Tab. I), dépend du niveau de contamination des échantillons mais les différences de répétabilité observées entre les niveaux de contamination sont, avec le BactoScan FC, fortement atténuées. Nettement meilleure que celle du BactoScan 8000 pour des niveaux de contamination inférieurs à $100000 \mathrm{UFC} \cdot \mathrm{mL}^{-1}$, c'est-à-dire aux niveaux les plus défavorables, la répétabilité du BactoScan FC est moins bonne pour des niveaux de contamination plus élevés. Elle reste néanmoins meilleure que celle de la méthode de référence (Tab. I), excepté pour des niveaux de contamination très faibles $\left(<10000 \mathrm{UFC} \cdot \mathrm{mL}^{-1}\right)$.

La stabilité de l'appareil a été évaluée à court terme $(6 \mathrm{~h})$ et à long terme (10 semaines). Les valeurs de reproductibilité à court terme
(Tab. II) sont, pour les niveaux de contamination $>10000 \mathrm{UFC} \cdot \mathrm{mL}^{-1}$, plus faibles que celles citées par Trossat et al. [11] pour le même modèle de BactoScan $\left(\mathrm{GRSD}_{\mathrm{R}}\right.$ compris entre 6,41 et $8,90 \%$ ). Les valeurs de reproductibilité à long terme (Tab. III) sont, par contre, plus élevées que celle obtenue par Trossat et al. [11] avec un même type d'échantillons stabilisés et un niveau de contamination comparable $\left(\mathrm{GRSD}_{\mathrm{R}} 2 \%\right)$. Comparé au BactoScan 8000, le BactoScan FC a une reproductibilité intra-jour plus faible pour des niveaux de contamination inférieurs à $100000 \mathrm{UFC} \cdot \mathrm{mL}^{-1}$. Dasen et al.

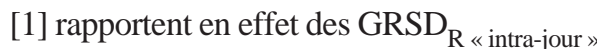
de $13 \%$ et $22 \%$ pour des niveaux de contamination de, respectivement, 30000 et $72000 \mathrm{UFC} \cdot \mathrm{mL}^{-1}$. Ce n'est pas le cas pour un niveau de contamination élevé : le $\mathrm{GRSD}_{\mathrm{R} \text { «intra-jour » }}$ du BactoScan 8000 est de $1,6 \%$ seulement pour un niveau de contamination de $290000 \mathrm{UFC} \cdot \mathrm{mL}^{-1}$ [1]. Il est à noter que, pour cette étude, les échantillons étaient stabilisés par des agents conservateurs, ce qui améliore la reproductrbilité, surtout en cas de fortes contaminations. Avec un conservateur, le $\mathrm{GRSD}_{\mathrm{R}}$ d'échantillons ayant environ $250000 \mathrm{UFC} \cdot \mathrm{mL}^{-1}$ passe en effet de 8,90\% à 5,20\% [11]. À long terme, pour un niveau de contamination comparable (72 $000 \mathrm{UFC} \cdot \mathrm{mL}^{-1}$ ), le BactoScan FC a une reproductibilité plus faible que le BactoScan $8000: \mathrm{GRSD}_{\mathrm{R}}$ « entre-jours » de $22 \%$ [1].

La relation entre le BactoScan FC et la méthode de référence est linéaire sur toute la gamme de contaminations analysée ; depuis les valeurs les plus petites $\left(\simeq 700 \mathrm{UFC} \cdot \mathrm{mL}^{-1}\right)$ jusqu' aux valeurs les plus élevées $\left(=26200000 \mathrm{UFC} \cdot \mathrm{mL}^{-1}\right)$. Le seuil de détermination du BactoScan FC n'a pu être établi avec précision. L'analyse de la relation a toutefois permis d'estimer qu'il est inférieur à $4000 \mathrm{UFC} \cdot \mathrm{mL}^{-1}$. Le seuil de détermination du BactoScan FC est inférieur à celui du BactoScan 8000 qui se situe entre moins de 10000 et $25000 \mathrm{UFC} \cdot \mathrm{mL}^{-1}$ $[1,9]$. 
La justesse de l'estimation de la valeur de référence $\left(\mathrm{s}_{\mathrm{y}, \mathrm{x}}\right)$ est de 0,287 $\log _{10}$ $\mathrm{UFC} \cdot \mathrm{mL}^{-1}$. C'est une valeur comparable à la valeur de $0,301 \log _{10} \mathrm{UFC} \cdot \mathrm{mL}^{-1}$ trouvée lors de l'évaluation du BactoScan FC par Trossat et al. [11]. Elle se situe dans l'ordre de grandeur des écarts-types généralement trouvés, pour ce type de relation, avec les versions antérieures du BactoScan : 0,296 ; 0,$23 ; 0,31 ; 0,277$ et $0,30[1,2,8,9,12]$.

\section{CONCLUSION}

L'utilisation d'une méthode alternative à la méthode de référence pour le dénombrement des germes en routine est acceptée dans de nombreux pays européens, notamment en Belgique. Il ne s'agit dès lors pas d'évaluer la pertinence de remplacer la méthode de référence par une méthode de routine mais bien la capacité d'un nouveau modèle de BactoScan, le FC, à remplacer les systèmes admis et plus particulièrement le BactoScan 8000 qui est la méthode alternative la plus représentée en Belgique.

Le BactoScan FC, d'utilisation simplifiée, présente des caractéristiques instrumentales et analytiques au moins équivalentes à celles du BactoScan 8000, excepté pour la répétabilité de l'analyse d'échantillons fortement contaminés (plus de $100000 \mathrm{UFC} \cdot \mathrm{mL}^{-1}$ ). Une nette amélioration de la répétabilité et de la reproductibilité dans le temps a été observée pour l'analyse d'échantillons faiblement contaminés (moins de $100000 \mathrm{UFC} \cdot \mathrm{mL}^{-1}$ ). Il offre en outre l'avantage de pouvoir déterminer des teneurs en germes très faibles, ce qui constitue un atout certain au vu de l'amélioration croissante de la qualité du lait cru.

Cette étude ayant été réalisée dans les conditions de la pratique, avec les échantillons de lait analysés par le laboratoire interprofessionnel, on peut conclure que le BactoScan FC présente les caractéristiques requises pour être utilisé dans le cadre du paiement du lait.

\section{REMERCIEMENTS}

Les auteurs remercient le personnel du laboratoire interprofessionnel de Battice (Liège), où est installé le BactoScan FC, pour l'accueil qu'il leur a réservé.

\section{RÉFÉRENCES}

[1] Dasen A., Olid R.M., Piton-Malleret C. Grappin R., Evaluation du BactoScan 8000 pour la numération automatique et rapide de la flore microbienne du lait cru, Lait 71 (1991) 661-670.

[2] Grappin R., Dasen A., Favennec P., Numération automatique et rapide des bactéries du lait cru à l'aide du BactoScan, Lait 65 (1985) 123-147.

[3] International Dairy Federation, Definition and evaluation of the overall accuracy of indirect methods of milk analysis, FIL-IDF standard 128, Brussels, Belgium, 1985.

[4] International Dairy Federation, Enumeration of microorganisms, FIL-IDF standard 100B, Brussels, Belgium, 1991.

[5] International Dairy Federation, Precision characteristics of analytical methods, FIL-IDF standard 135B, Brussels, Belgium, 1991.

[6] International Dairy Federation, Quantitative determination of bacteriological quality, FILIDF standard 161A, Brussels, Belgium, 1995.

[7] International Dairy Federation, Preparation of samples and dilutions for microbiological examination, FIL-IDF standard 122C, Brussels, Belgium, 1996.

[8] Nieuwenhof F.F.J., Hoolwerf J.D., Suitability of Bactoscan for the estimation of the bacteriological quality of raw milk, Milchwissenschaft 43 (1988) 577-586.

[9] Suhren G., Reichmuth J., Determination of the bacteriological quality of raw milk by automatic fluorescent microscopic counting of single bacteria (BactoScan 8000) - a review, Kiel. Milchwirtsch. Forschungsber. 49 (1997) 163-186.

[10] Suhren G., Walte H.-G., First experiences with automatic flow cytometric determination of total bacterial count in raw milk, Kiel. Milchwirtsch. Forschungsber. 50 (1998) 249-275.

[11] Trossat Ph., Leray O., Rollier P., Rapport préliminaire d'évaluation du Bactoscan FC, Ceca Lait, Poligny, 1998.

[12] van Heddeghem A., Reybroeck W., van Crombrugge J., De bruikbaarheid van de Bactoscan 2/87 bij de bepaling van het kiemgetal van rauwe melk, Rev. Agric. 43 (1990) 1041-1049. 
